\title{
Conocimiento y prácticas relacionadas con control y prevención de COVID-19 en trabajadores de la salud
}

\author{
Knowledge and practice regarding COVID-19 infection, control and prevention \\ among healthcare workers
}

\author{
Herberth G. Maldonado Briones ${ }^{1,2,3}$, Mario A. Melgar Toledo ${ }^{3,4,5}$, \\ Nancy V. Sandoval Paiz ${ }^{5,6}$ \\ ${ }^{1}$ Centro de Estudios en Salud, Universidad del Valle de Guatemala, ${ }^{2}$ Unidad de Cirugía Cardiovascular de Guatemala, \\ ${ }^{3}$ Facultad de Ciencias Médicas, Universidad San Carlos de Guatemala, ${ }^{4}$ Unidad de Oncología Pediátrica, Guatemala, \\ ${ }^{5}$ Hospital Roosevelt, Guatemala ${ }^{6}$ Facultad de Medicina, Universidad Rafael Landívar, Guatemala
}

*Autor al que se dirige la correspondencia: hmaldonado@ces.uvg.edu.gt

Recibido: 20 de julio 2019 / Revisión: 17 de agosto 2020 / Aceptado: 15 de octubre 2020

\section{Resumen}

T a enfermedad por coronavirus 2019 (COVID-19) ha afectado a los trabajadores de la salud (TS) quienes suLman riesgo de exposición en la comunidad y el trabajo. El conocimiento y preparación son fundamentales, sin embargo, durante la pandemia se han suspendido las actividades presenciales de formación-capacitación. El objetivo de este estudio fue determinar si la educación en línea genera una diferencia en el conocimiento y práctica de prevención y control de infecciones (PCI) para la COVID-19 en TS. Previo consentimiento, se administró un cuestionario antes-después a los participantes de un curso de PCI en línea. Para la evaluación de conocimientos y prácticas se diseñaron preguntas tipo Likert con valores de 1 a 5 , realizando el análisis con la prueba no paramétrica de rangos con signo de Wilcoxon para muestras relacionadas. En 345 participantes, la mediana fue 30 (RIC 15) años, femenino (224, 65\%), residentes en el departamento de Guatemala (221, 57\%), labora en sector público $(155,44.9 \%)$, sector privado $(154,44.6 \%)$, y seguro social $(29,8.4 \%)$. La evaluación antes-después mostró diferencia significativa de la brecha existente en el conocimiento y las prácticas de PCI ( $<$ < .05). La mayor brecha se observó en el autocuidado de la salud física, mental y nutricional. En las percepciones, uno de cada tres encuestados manifestó temor a sufrir estigma en caso de resultar infectados. La educación y entrenamiento en PCI es esencial ante una enfermedad altamente contagiosa que amenaza la salud y seguridad de los TS, principalmente en entornos sanitarios con recursos limitados.

Palabras claves: Actitudes, COVID-19, trabajadores de la salud, conocimiento, prevención y control de infecciones

\section{Abstract}

$\mathrm{T}$ he coronavirus disease 2019 (COVID-19) has affected health workers (HCWs) who adds risk of exposure in the community and at work. Knowledge and preparation are essential. However, during the pandemic, face-to-face training activities have been suspended. The objective of this study was to determine if online education generate a difference in the knowledge and practice of infection prevention and control (IPC) of COVID-19 in HCWs. With prior consent, a before-after questionnaire was administered to participants of an online PCI course. For the evaluation of knowledge and practices, Likert-type questions were designed with values from 1 to 5 , performing the analysis with the non-parametric test of Wilcoxon signed ranges for related samples. In 345 participants, the median was 30 (IQR 15) years, female (224, 65\%), living in the department of Guatemala (221, 57\%), working in the public sector $(155,44.9 \%)$, private sector $(154,44.6 \%)$, and social security $(29,8.4 \%)$. The before-after assessment showed a significant difference in the gap between knowledge and practices. The largest gap was observed in self-care of physical, mental, and nutritional health. About perceptions, one out of every three respondents expressed fear of suffering stigma if they get infected. Education and training in IPC are essential in the face of a highly contagious disease that threatens the health and safety of HCWs, mainly in healthcare settings with limited resources.

Keywords: Attitudes, COVID-19, health workers, knowledge, prevention and infection control 


\section{Introducción}

Al 19 de julio de 2020 ( 8 meses después de iniciada la pandemia) la enfermedad por coronavirus 2019 (COVID-19) ha provocado más de 14 millones de casos y poco más de medio millón de muertes (World Health Organization [WHO], 2020). En Guatemala, se reportan a la misma fecha poco más de 38 mil casos y $1 \mathrm{mil}$ 485 muertes (letalidad de 3.8\%), la curva epidémica aún está en ascenso y se estima que su pico se alcanzará a mitad o finales de agosto de 2020 (Ministerio de Salud Pública y Asistencia Social [MSPAS], 2020).

Para interrumpir la transmisión se ha recomendado a la población permanecer en casa, sin embargo, los trabajadores de la salud (TS) deben prestar servicios de forma continua, e incluso incrementar su carga de trabajo por la reducción de fuerza laboral por contagios masivos (Barranco \& Ventura, 2020). Así, los TS tienen mayor riesgo de infección y transmisión pues tendrán contacto frecuente con portadores asintomáticos e individuos pre-sintomáticos y sintomáticos, infectados por SARS-CoV-2, siendo el riesgo mucho mayor cuando existe limitación o carencias para acceder al equipo de protección personal (EPP) (Barrett et al., 2020; Belingheri et al., 2020; Nguyen et al., 2020).

Existen datos limitados sobre si los TS con la COVID-19 tienen mayor mortalidad y complicaciones que la población general (Wander et al., 2020). En un reporte de Estados Unidos, uno de cada cuatro trabajadores infectados presentó múltiples factores de riesgo para enfermedad severa (Gibson \& Greene, 2020). Es conocido que los TS tienen una alta prevalencia de obesidad y de patologías crónicas no transmisibles como diabetes e hipertensión arterial. Adicionalmente, los TS suelen adherirse a estilos de vida poco saludables como el sedentarismo, la mala alimentación y el estrés laboral (Villarreal, 2003).

Durante la pandemia los TS han sido vulnerables al contagio, reconociéndose un subregistro en el número de casos y muertes reportadas (Kursumovic et al., 2020). Mientras la prevalencia en países de elevados recursos económicos como España y Estados Unidos ha sido del $24 \%$ y del $19 \%$ respectivamente (Centers for Disease Control and Prevention [CDC], 2020; Red Nacional de Vigilancia Epidemiológica [Renave], 2020), en países de bajos y medianos ingresos los datos sobre casos y muertes en TS son escasos y limitados a reportes de noticias. En nuestro país, una nota reciente de elPeriódico de fecha 7 de julio 2020 reporta 586 infecciones en trabajadores de hospitales nacionales por el Ministerio de Salud Pública y Asistencia Social con ocho fallecimientos (. Lamentablemente se espera que el número de las muertes se incrementen según avance la pandemia, por lo que se debe considerar proteger a los médicos mayores de 60 años y aquellos con enfermedades crónicas del trabajo de primera línea (Ashcroft, 2020).

Entre las razones principales para el contagio de TS durante la atención sanitaria están la falta de medidas institucionales de control de infecciones, falta de conocimiento y preparación en la etapa inicial del brote, especialmente para la colocación y retiro de EPP (Xiao et al., 2020). La falta de conocimientos en TS influye en sus actitudes y prácticas, que si son incorrectas incrementan el riesgo de infección (Wu \& McGoogan, 2020).

Ante esta amenaza para los TS la preparación es prioritaria, pero paradójicamente las capacitaciones y entrenamientos presenciales fueron suspendidos para reducir los contagios. Como respuesta, se han organizado seminarios y cursos en línea para fortalecer el conocimiento y competencias ante esta nueva enfermedad, entre ellas aspectos relacionados a la prevención y control de infecciones (PCI) enfocado en la COVID-19. La enseñanza para TS en línea es una estrategia relativamente nueva, pero su uso se ha incrementado durante la pandemia.

El objetivo de este estudio fue evaluar si la educación en línea a través de un curso enfocado en PCI para TS produce una mejora en sus conocimientos y prácticas sobre la enfermedad.

\section{Materiales y métodos}

Se realizó una encuesta transversal a TS en Guatemala antes y después de tomar el Curso en línea sobre Prevención, Control y Manejo de la COVID-19 para personal de salud en Guatemala facilitado por la Asociación Guatemalteca de Enfermedades Infecciosas (AGEI, 2020) con la colaboración de la oficina local de la Organización Panamericana de la Salud y de la Oficina Regional para Centroamérica de los Centros para el Control y Prevención de Enfermedades (CDCCAR) de los Estados Unidos.

El cuestionario fue diseñado y validado con un grupo pequeño de $39 \mathrm{TS}$. Se administró en idioma español utilizando formularios de Google, una herramienta en línea gratuita para el diseño, recolección y almacenamiento de datos de cuestionarios. Se invitó a la participación voluntaria durante la inscripción y 
al finalizar el curso. El enlace para la participación en el curso fue distribuido por correo electrónico y a través de redes sociales de AGEI en las plataformas Facebook y Twitter. La recolección de datos para la evaluación antes se realizó del 7 al 10 de abril de 2020 hasta la medianoche. Al finalizar el curso se envió por correo electrónico un enlace con el cuestionario después, finalizando la recepción de respuestas el 30 de junio de 2020.

En el cuestionario antes se solicitó información sobre demografía, sector laboral, tipo de especialidad, padecimientos crónicos y hábito de fumar. En los cuestionarios antes-después se realizaron preguntas de autoevaluación de conocimientos y prácticas de $\mathrm{PCI}$ con enfoque en la COVID-19 utilizando una escala de Likert de 1 a 5. Los aspectos fueron evaluados de acuerdo a las competencias básicas para el control de infecciones del European Centre for Disease Prevention and Control (ECDC, 2013). Se realizó una evaluación de actitudes y percepción de riesgo utilizando preguntas cerradas.

Los datos crudos sin identificadores fueron exportados a un archivo de formato XLS. Se eliminaron registros duplicados y respuestas en blanco. Se parearon las respuestas colectadas utilizando como identificador el correo electrónico. El análisis estadístico se realizó utilizando el software RStudio ${ }^{\circledR}$ utilizando los paquetes estadísticos Likert, HH y coin. Se utilizó estadística descriptiva para describir las características de los participantes. Para la evaluación antes-después de los componentes de conocimiento y práctica, se sumaron los puntajes de cada participante y posteriormente se aplicó la prueba no paramétrica de rangos con signo de Wilcoxon para muestras relacionadas, considerando un resultado significativo $<.05$.

\section{Resultados}

Se registraron en el curso 2,551 personas, de las cuales 2,290 proporcionaron consentimiento y respondieron el cuestionario antes. El cuestionario después fue respondido por 451 personas, emparejando las respuestas antes-después para 345 sujetos que finalmente fueron incluidos en el análisis. Se presentaron las características de los participantes y posteriormente los resultados del análisis comparativo pareado antes-des- pués de los aspectos sobre conocimiento y prácticas de PCI.

\section{Datos demográficos}

La mediana de edad fue de 30 años (RIC 15), predominio del sexo femenino $(224,65 \%)$, residentes de los departamentos de Guatemala 58\%, Quetzaltenango 7\%, Sacatepéquez 6\%, Alta Verapaz, Chiquimula, Escuintla y Huehuetenango 3\% cada uno, Sololá, Totonicapán y Zacapa cada uno $2 \%$, el resto de departamentos suman un $8.6 \%$.

\section{Lugar de trabajo, nivel de atención y especialidad}

Del total de participantes, 155 (44.9\%) laboran para el MSPAS, 154 (44.6\%) en el sector privado, 29 $(8.4 \%)$ en el Instituto Guatemalteco de Seguridad Social (IGSS) y 7 (2\%) labora para al menos dos de las anteriores categorías. La prestación de servicios por nivel de atención fue de 168 (48.7\%) para tercer nivel (hospitales, sanatorio), 41 (12\%) para segundo nivel (centros de salud y clínicas especializadas) y $136(39 \%)$ en nivel primario (puestos de salud, clínicas ambulatorias y otros servicios). La profesión reportada fue medicina en 294 (85.2\%), enfermería 40 (11.5\%) y afines $11(3 \%)$. La especialidad de práctica reportada en médicos fue medicina general 139 (47\%), ginecología y obstetricia $22(7 \%)$, medicina interna $19(6 \%)$, pediatría $15(5 \%)$, anestesiología, cirugía oral y maxilofacial, medicina de urgencias y oftalmología con $3 \%$ cada uno, otras especialidades $23 \%$.

\section{Factores de riesgo para enfermedad severa por la COVID-19}

De los participantes, 93 (27\%) reportaron al menos un factor de riesgo, $16(3.8 \%)$ reportaron dos factores y $6(1.7 \%)$ tres o más factores de riesgo. La prevalencia de factores reportados fueron edad mayor o igual a 60 años de edad en 14 (4\%), hábito de fumar $3 \%$, obesidad (IMC $>30$ ) en $31(9 \%)$, hipertensión arterial $28(8 \%)$, asma $25(7.2 \%)$, diabetes mellitus tipo 2 en $11(3.2 \%)$, inmunosupresión 11 (3.2\%). 


\section{Organización del lugar de trabajo para la pandemia: identificación de casos y aislamiento, EPP}

De los participantes, 272 (79\%) respondieron que existe un comité dirigiendo las acciones de PCI en su lugar de trabajo, mientras que 218 (89\%) indicaron que existen procedimientos para identificación y aislamiento de casos, pero solo 197 (57\%) considera que son apropiados. En relación al suministro de EPP esencial en el lugar de trabajo, el 97 (28\%) manifestaron que le proporcionan el EPP regularmente y 93 (27\%) no lo recibe.

\section{Capacitación previa en precauciones estándar}

Entre los participantes, 169 (49\%) había recibido durante el último año un curso formal de higiene de manos y 118 (34.2\%) habían recibido capacitación para el uso de EPP.

\section{Conocimiento sobre control y prevención de la COVID-19}

La mediana de la suma de puntajes en conocimiento obtenidos por los participantes antes del curso fue de 23 (RIC 6) y después del curso 30 (RIC 2), siendo el incremento estadísticamente significativo $(p<.001)$. Los resultados y contenidos del conocimiento evaluados están resumidos en la Gráfica 1.

\section{Prácticas de control y prevención de la COVID-19}

La mediana de la suma de los puntajes en práctica obtenidos por los participantes antes del curso fue de 16 (RIC 4) y después del curso 20 (RIC 1), siendo el incremento estadísticamente significativo $(p<.001)$. Los resultados y contenidos de práctica evaluados están resumidos en la Gráfica 2.

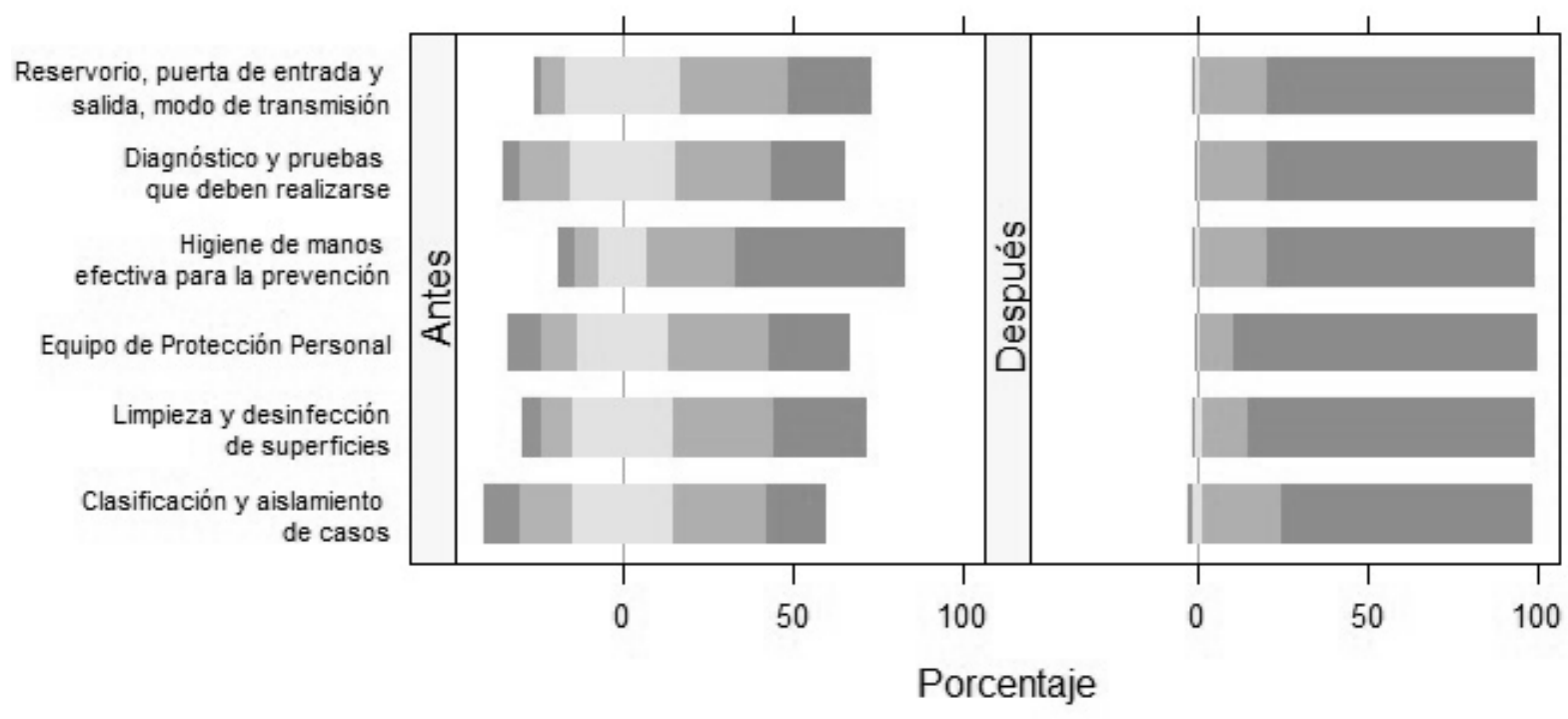

Totalmente en desacuerdo
En desacuerdo
Neutral

De acuerdo

Totalmente de acuerdo

Figura 1. Gráfico comparativo de distribución de respuestas sobre conocimiento de prevención y control de COVID19 en trabajadores de salud antes y después del curso. 


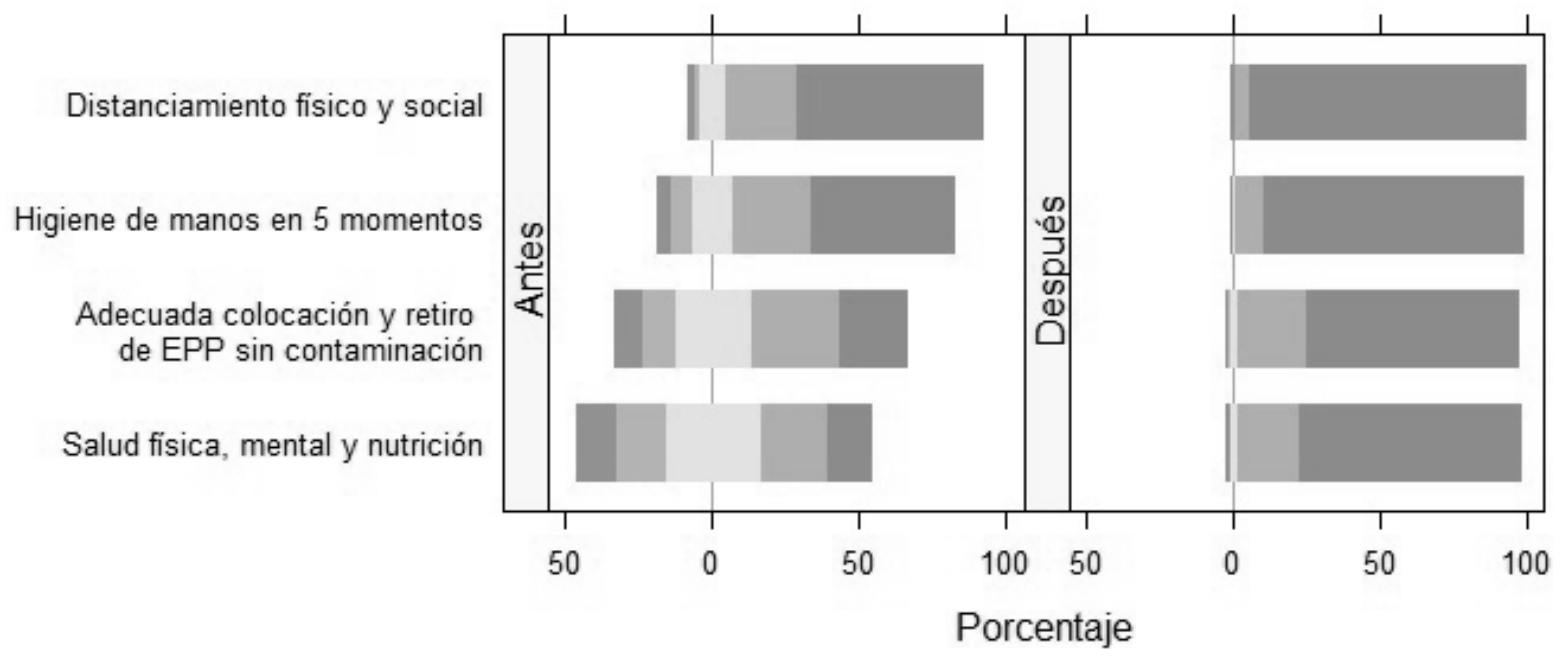

Totalmente en desacuerdo
En desacuerdo
Neutral

De acuerdo

Totalmente de acuerdo

Figura 2. Gráfico comparativo de distribución de respuestas sobre prácticas de prevención y control de COVID-19 en trabajadores de salud antes y después del curso.

\section{Actitud y percepción de riesgo frente a la COVID-19}

Uno de cada tres participantes manifestó temor a ser estigmatizado por proporcionar atención directa a pacientes confirmados con COVID-19 y uno de cada cinco percibe riesgo si un compañero de labores proporciona atención a pacientes confirmados con COVID-19. Los resultados se resumen en la Tabla 1.

\section{Discusión}

Los resultados de este estudio muestran brechas entre los TS en algunos aspectos de conocimiento y prácticas de $\mathrm{PCI}$, así como una importante percepción de riesgo y temor a la infección. Tres de cada diez respondedores indicaron padecer al menos un factor de riesgo asociado a sufrir enfermedad severa, siendo los más frecuentes la obesidad, hipertensión arterial y el asma.

Por la otra parte, la evaluación antes-después evidenció una diferencia significativa en el conocimiento y prácticas de PCI, lo que soporta la hipótesis planteada de que la educación en línea es una alternativa efectiva para fortalecer las competencias de TS. Esto alienta a continuar los esfuerzos de entrenamiento a distancia en un período donde la distancia física y la educación son esenciales (Escalera-Antezana et al., 2020). Sin embargo, la educación y el entrenamiento deben ser reforzados, pues se ha observado que mientras la pandemia avanza, los TS que laboran en áreas de alto riesgo con casos confirmados, mejoran su desempeño, sin embargo, aquellos que laboran en áreas de bajo riesgo, muestran conductas inapropiadas, resultando en uso excesivo de insumos y recurso humano (Lai et al., 2020).

En India, un estudio en 453 TS, mostró que el conocimiento sobre transmisión fue del $61 \%$, siendo las redes sociales la principal fuente de información, encontrando una asociación entre edad, profesión y pobre conocimiento (Bhagavathula et al., 2020). En el actual estudio se encontró algo similar, debido a que 195/345 (57\%) manifestó estar de acuerdo o totalmente de acuerdo en tener conocimientos sobre la transmisión. Este conocimiento es esencial y debe actualizarse periódicamente de acuerdo a evidencia nueva que permita modificar las prácticas de prevención (Sommerstein et al., 2020). 
Tabla 1

Evaluación de actitudes y percepción de riesgo a COVID-19 en TS

\begin{tabular}{llll}
\hline Pregunta & Si (\%) & No (\%) & Tal vez (\%) \\
\hline $\begin{array}{l}\text { ¿Se siente vulnerable a infectarse al salir de la casa? } \\
\begin{array}{l}\text { ¿Se sentiría vulnerable si sabe que un colega del hospital ha estado } \\
\text { atendiendo casos de COVID-19 }\end{array}\end{array}$ & $10(3)$ & $250(73)$ & $84(24)$ \\
$\begin{array}{l}\text { ¿Le preocupa ser enviado a cuarentena en un hospital de referencia del } \\
\text { Ministerio de Salud pública? }\end{array}$ & $101(29)$ & $144(42)$ & $122(36)$ \\
$\begin{array}{l}\text { ¿Se sentiría amenazado si sus colegas se enteraran de que se ha expuesto a } \\
\text { un caso de COVID-19, aunque haya usado EPP apropiadamente? }\end{array}$ & $109(32)$ & $101(29)$ & $133(39)$ \\
\hline
\end{tabular}

La higiene de manos es la piedra angular de la prevención de la transmisión en la atención sanitaria y aunque el 90\% (312/345) aseguró tener conocimiento sobre higiene de manos, el 74\% (256/345) indicó que aplica el concepto de "mis cinco momentos para la higiene de las manos" (antes y después de tocar al paciente, antes de una tarea aséptica, después de la exposición a fluidos corporales y después del contacto con el entorno sanitario). Otro aspecto fundamental para evitar el contagio es el uso de EPP, específicamente mascarillas y respiradores N95, batas impermeables, guantes y protección ocular. El 69\% (240/345) manifestó tener conocimiento sobre EPP, pero solo la mitad indicó saber como colocar y retirarlo apropiadamente. Estos hallazgos no son sorprendentes, ya que menos de la mitad reportaron haber recibido de forma reciente un curso de higiene de manos y EPP, aspectos que deben ser fortalecidos con regularidad.

Otro hallazgo relevante en este estudio fue la percepción de riesgo a infectarse en la comunidad y a través de compañeros de trabajo que estén expuestos a la COVID-19, así como preocupación a sufrir estigma y vulnerabilidad en caso de proporcionar atención a pacientes confirmados. Esto se ha visto en un estudio similar con 407 TS en Egipto, que reportó que un alto nivel de conocimiento hacia la COVID-19 se correlacionó de forma positiva con la actitud, donde el $83 \%$ de los participantes reportó su temor a infectarse y a sufrir estigma social si fuese infectado (Wahed et al., 2020).

La salud mental de los TS debe ser protegida durante la pandemia y pasar del aforismo "sanate a ti mismo" al de "sanar al sanador" (Wong et al., 2020). $\mathrm{El}$ aspecto con la mayor brecha identificada fue el de autocuidado de la salud física, mental y nutricional. Se ha demostrado que el cuidado personal mejora el rendimiento de los TS al reducir el agotamiento, el trauma indirecto, la fatiga por compasión y otros problemas psicológicos relacionados con el estrés (Smith, 2017).

Las limitaciones de este estudio incluyen la naturaleza del diseño de un estudio transversal distribuido en línea durante un reporte alarmante de casos a nivel mundial. Las respuestas son auto reportadas, por lo que dependen de la honestidad y sinceridad del sujeto, por tanto, sujeto a sesgo. El estudio no alcanza una representatividad de participantes a nivel geográfico, nivel de atención y con una pequeña representación de la profesión de enfermería y otras profesiones afines. Aunque el estudio es considerado de riesgo mínimo para los participantes, no fue factible una aprobación ética debido a las restricciones de ese momento. Futuros estudios deben incluir un diseño longitudinal que considere una mayor representatividad de los TS en los aspectos mencionados para poder generalizar los resultados. A pesar de estas limitaciones, los hallazgos proveen información relevante sobre el impacto en la mejora del conocimiento y prácticas de prevención y control frente a la COVID-19 luego de un curso en línea.

La COVID-19 ha puesto en evidencia las debilidades de los sistemas de salud latinoamericanos que, debido a la falta de políticas y acciones preventivas son incapaces de evitar la transmisión sostenida de enfermedades (Delgado et al., 2020; Kalra et al., 2020). El final de la pandemia con el menor número de infectados y muertos entre los TS solo será posible con una adecuada prevención de riesgos laborales, así como un 
seguimiento de los efectos en la salud física y mental que ocasionan los cambios de horario, el estrés y el duelo continuo por la pérdida de amigos, compañeros y familiares (Benavides, 2020). Es urgente la acción de las autoridades de la salud pública y privadas para la gestión e implementación de programas de PCI, así como la promoción y recuperación de la salud física y mental en sus trabajadores.

\section{Agradecimientos}

Agradecemos a los participantes del curso y de este estudio. Por el apoyo para la realización del curso a Eswin Romero y Ana Lucía Campos, a los miembros de la AGEI, especialmente Alicia Chang, Carlos Grazioso, Claudio Ramírez, Estuardo Tercero, Verónica Gómez, a la oficina de la OPS/OMS en Guatemala, especialmente a Oscar Barreneche y Mónica Guardó, a la oficina de CDC-CAR, especialmente a Emily Zielinski y Andrés Espinosa-Bode. Este trabajo no recibió ningún financiamiento.

\section{Referencias}

Asociación Guatemalteca de Enfermedades Infecciosas. (2020). Curso en línea sobre Prevención, Control y Manejo de COVID-19 para personal de salud en Guatemala. Recuperado de https://bit.ly/2DGjibh

Ashcroft, J. (2020). Keep older healthcare workers off the covid-19 front line. British Medical Journal, 369, m1511. https://doi.org/10.1136/bmj.m1511

Barranco, R., \& Ventura, F. (2020). Covid-19 and infection in healthcare workers: An emerging problem. Medico-Legal Journal, 88(2), 65-66. https://doi.org/10.1177/0025817220923694

Barrett, E. S., Horton, D. B., Roy, J., Gennaro, M. L., Brooks, A., Tischfield, J., . . Panettieri, R. A. (2020). Prevalence of SARS-CoV-2 infection in previously undiagnosed health care workers at the onset of the U.S. COVID-19 epidemic. medRxiv. https://doi.org/10.1101/2020.04.20.20072470

Belingheri, M., Paladino, M. E., \& Riva, M. A. (2020). Beyond the assistance: Additional exposure situations to COVID-19 for healthcare workers. The Journal of Hospital Infection, 105(2), 353. https://doi.org/10.1016/j.jhin.2020.03.033
Benavides, F. G. (2020). La salud de los trabajadores y la COVID-19. Archivos de Prevención de Riesgos Laborales, 23, 154-158. https://doi.org/10.12961/ aprl.2020.23.02.02

Bhagavathula, A. S., Aldhaleei, W. A., Rahmani, J., Mahabadi, M. A., \& Bandari, D. K. (2020). Knowledge and perceptions of COVID-19 among health care workers: Cross-sectional study. Journal of Medical Internet Research Public Health and Surveillance, 6(2), e19160. https:// doi.org/10.2196/19160

Centers for Disease Control and Prevention. (2020). Characteristics of health care personnel with COVID-19 - United States, February 12-April 9, 2020. Morbidity and Mortalilty Weekly Report, 69(15), 477-481. https://doi.org/10.15585/mmwr. $\mathrm{mm} 6915 \mathrm{e} 6$

Delgado, D., Wyss Quintana, F., Perez, G., Sosa Liprandi, A., Ponte-Negretti, C., Mendoza, I., \& Baranchuk, A. (2020). Personal safety during the COVID-19 pandemic: Realities and perspectives of healthcare workers in Latin America. International Journal of Environmental Research and Public Health, 17(8), 2798. http:// doi.org/10.3390/ijerph17082798

European Centre for Disease Prevention and Control. (2013). Core competencies for infection control and hospital hygiene professionals in the European Union. Stockholm: Autor. Recuperado de https://healthmanagement.org/c/hospital/ issuearticle/core-competencies-for-infectioncontrol-and-hospital-hygiene-professionals-inthe-european-union

elPeriódico. (07 de julio de 2020). Más de 500 salubristas han padecido COVID-19. Recuperado de https://elperiodico.com.gt/nacion/2020/07/07/ mas-de-500-salubristas-han-padecido-covid-19/

Escalera-Antezana, J. P., Cerruto-Zelaya, P. E., Apaza-Huasco, M., Miranda-Rojas, S. H., Flores-Cardenas, C. A., Rivera-Zabala, L., . . . Rodriguez-Morales, A. J. (2020). Healthcare workers' and students' knowledge regarding the transmission, epidemiology and symptoms of COVID-19 in 41 cities of Bolivia and Colombia. Travel Medicine and Infectious Disease, 37, Article e101702. https://doi.org/10.1016/j. tmaid.2020.101702 
Red Nacional de Vigilancia Epidemiológica. (2020). Análisis de los casos de COVID-19 en personal sanitario notificados a la RENAVE hasta el 10 de mayo en España. Recuperado de https://www.isciii.es/QueHacemos/ Servicios/VigilanciaSaludPublicaRENAVE/ EnfermedadesTransmisibles/Documents/ INFOR MES/Informes\%2 0 COVID-19/ COVID-19\%20en\%20personal\%20sanitario\%20 29\%20de\%20mayo\%20de\%202020.pdf

Gibson, D. M., \& Greene, J. (2020). Risk for severe COVID-19 illness among health care workers who work directly with patients. Journal of General Internal Medicine, 35(9), 2804-2806. https://doi.org/10.1007/s11606-020-05992-y

Kalra, A., Michos, E. D., \& Chinnaiyan, K. M. (2020). COVID-19 and the healthcare workers. European Heart Journal, 41(31), 2936-2937. https://doi. org/10.1093/eurheartj/ehaa489

Kursumovic, E., Lennane, S., \& Cook, T. M. (2020). Deaths in healthcare workers due to COVID-19: The need for robust data and analysis. Anaesthesia, 75(8), 989-992. https:// doi.org/10.1111/anae.15116

Lai, X., Wang, X., Yang, Q., Xu, X., Tang, Y., Liu, C., ... Chen, H. (2020). Will healthcare workers improve infection prevention and control behaviors as COVID-19 risk emerges and increases, in China? Antimicrobial Resistance and Infection Control, 9(1), 83. https://doi. org/10.1186/s13756-020-00746-1

Ministerio de Salud Pública y Asistencia Social. (19 de julio de 2020). Situación de COVID-19 en Guatemala. Disponible en https://tablerocovid. mspas.gob.gt/

Nguyen, L. H., Drew, D. A., Joshi, A. D., Guo, C. G., Ma, W., Mehta, R. S., . . Chan, A. T. (2020). Risk of COVID-19 among frontline healthcare workers and the general community: a prospective cohort study. medRxiv. https://doi. org/10.1101/2020.04.29.20084111

Smith, K. L. (2017). Self-care practices and the professional self. Journal of Social Work in Disability \& Rehabilitation, 16(3-4), 186-203. https://doi.org/10.1080/1536710X.2017.1372236

Sommerstein, R., Fux, C. A., Vuichard-Gysin, D., Abbas, M., Marschall, J., Balmelli, C., . . Swissnoso. (2020). Risk of SARS-CoV-2 transmission by aerosols, the rational use of masks, and protection of healthcare workers from COVID-19. Antimicrobial Resistance \& Infection Control, 9(1), 100. https://doi.org/10.1186/s13756020-00763-0

Villarreal, S. M. (2003). Prevalencia de la obesidad, patologías crónicas no transmisibles asociadas y su relación con el estrés, hábitos alimentarios y actividad física en los trabajadores del Hospital de la Anexión. Revistas de Ciencias Administrativas y Financieras de la Seguridad Social, 11(1), 83-96. Recuperado de http://www.scielo.sa.cr/ scielo.php?script $=$ sci_arttext\&pid=S1409$12592003000100009 \& \mathrm{nrm}=$ iso

Wahed, W. Y., Hefzy, E. M., Ahmed, M. I., \& Hamed, N. S. (2020). Assessment of knowledge, attitudes, and perception of health care workers regarding COVID-19, A cross-sectional study from Egypt. Journal of Community Health, 1-10. https://doi. org/10.1007/s10900-020-00882-0

Wander, P. L., Orlov, M., Merel, S. E., \& Enquobahrie, D. A. (2020). Risk factors for severe COVID-19 illness in healthcare workers: Too many unknowns. Infection Control \& Hospital Epidemiology, 1-2. https://doi.org/10.1017/ice.2020.178

World Health Organization. (2020). WHO Coronavirus Disease (COVID-19) Dashboard. Recuperado el 19 de julio del 2020, de https://covid19.who.int/

Wong, A. H., Pacella-LaBarbara, M. L., Ray, J. M., Ranney, M. L., \& Chang, B. P. (2020). Healing the healer: Protecting emergency health care workers' mental health during COVID-19. Annals of Emergency Medicine, 76(4), 379-384. https:// doi.org/10.1016/j.annemergmed.2020.04.041

Wu, Z., \& McGoogan, J. M. (2020). Characteristics of and important lessons from the coronavirus disease 2019 (COVID-19) outbreak in china: Summary of a report of 72314 cases from the Chinese Center for Disease Control and Prevention. Journal of the American Medical Association, 323(13), 1239-1242. https://doi. org/10.1001/jama.2020.2648

Xiao, J., Fang, M., Chen, Q., \& He, B. (2020). SARS, MERS and COVID-19 among healthcare workers: A narrative review. Journal of Infection and Public Health, 13(6), 843-848. https://doi. org/10.1016/j.jiph.2020.05.019 